\title{
Primary meningococcal arthritis in a COVID- 19 18-year-old man: a case report and review of the literature
}

\author{
Norman Ducatez ${ }^{1 *} \mathbb{D}$, Marine Chancel ${ }^{2}$, Youcef Douadi $^{1}$, Charles Dayen $^{1}$, Rémi Suguenot ${ }^{1}$, Emmanuelle Lecuyer $^{1}$, \\ Benoit Brihaye $^{2}$ and Houcine Bentayeb ${ }^{1}$
}

\begin{abstract}
Background: SARS-CoV-2 (severe acute respiratory syndrome coronavirus 2) infection is associated with various complications. PMA (primary meningococcal arthritis) is a rare meningococcus-associated disease causing arthritis of the knee usually, without any signs of invasive meningococcal disease. No case of PMA in a COVID-19 (coronavirus disease, 2019) patient has yet been described. PMA mainly strikes young adults. PMA is not associated with any immunocompromising condition. It has a better outcome than usual septic arthritis

Case presentation: Herein, we report an 18-year-old man diagnosed with COVID-19, later admitted with persistent fever, right knee arthralgia and maculopapular rash. Due to family history, psoriasis and Henoch-Schönlein purpura were hypothesized and ruled out. Finally, synovial fluid culture confirmed Neisseria meningitidis serogroup B arthritis without any other symptoms of invasive meningococcal disease. Healing was achieved quickly with surgery and antibiotics. We concluded in a PMA.
\end{abstract}

Conclusion: We describe here the first primary meningococcal arthritis in a COVID-19 patient and we hope to shine a light on this rare but serious complication.

Keywords: Arthritis, COVID-19, Neisseria meningitidis

\section{Background}

Worldwide, $10 \%$ of human beings are considered asymptomatic carriers of Neisseria meningitidis [1]. It can be a potentially aggressive bacteria, causing invasive and possibly deadly infections such as meningitis or septicemia $[1,2]$. Indeed it is known to cause fever, headache, nausea, vomiting, severe myalgias, nonspecific rash, sore throat and upper respiratory symptoms. Alongside the well-known meningitis and purpura fulminans, some presentations are far rarer, such as primary

\footnotetext{
*Correspondence: norman.ducatez@gmail.com

'Pneumology and Infectious Diseases department, Saint-Quentin hospital, 1 avenue Michel de l'hospital, 02100 Saint-Quentin, France

Full list of author information is available at the end of the article
}

meningococcal arthritis (PMA) and chronic meningococcemia [3]. Bloodstream dissemination mechanism is unknown.

In literature, PMA has three peaks of incidence, the early years of life, around the age of 20 and over the age of 65 , with the highest prevalence for the young adult peak [3]. The most common presentation is a monoarthritis of the knee, which is, by far, the most involved joint. The most found serogroup is N.meningitidis B [4]. No underlying condition, in particular no immunocompromising condition has been proved to facilitate PMA [3]. Lavoipierre et al. reported a case of a young woman with no medical history but with a family history of inflammatory rheumatism [5] that could indicate an underlying genetic susceptibility. Nonetheless some

C C The Author(s). 2021 Open Access This article is licensed under a Creative Commons Attribution 4.0 International License, which permits use, sharing, adaptation, distribution and reproduction in any medium or format, as long as you give appropriate credit to the original author(s) and the source, provide a link to the Creative Commons licence, and indicate if changes were made. The images or other third party material in this article are included in the article's Creative Commons licence, unless indicated otherwise in a credit line to the material. If material is not included in the article's Creative Commons licence and your intended use is not permitted by statutory regulation or exceeds the permitted use, you will need to obtain permission directly from the copyright holder. To view a copy of this licence, visit http://creativecommons.org/licenses/by/4.0/ The Creative Commons Public Domain Dedication waiver (http://creativecommons.org/publicdomain/zero/1.0/) applies to the data made available in this article, unless otherwise stated in a credit line to the data. 
similarities can be found in the reported cases, such as age, medical history, and clinical presentation. Young people, male or female [6] with no medical history are most represented [2-4], but we can also find several cases over the age of $60[3,7]$. The knee is very often involved, either as monoarthritis or as part of an oligoarthritis. The prognosis is generally good [3] unlike arthritis usually caused by Gram positive Cocci, (i.e. S.aureus or Streptococcus spp), or less often by Gram negative Bacilli. These infections are accompanied with morbidity, limited functional use of the impaired joints and reduced quality of life. Nowadays, it is acknowledged that a quick treatment, including surgical joint drainage and initial intravenous antibiotherapy reduces such consequences [2]. Up to 2019, only 47 cases of PMA have been reported in PubMed [3]. Finally, cases of acute arthritis during COVID-19 were reported [810] but none involving N.meningitidis.

Here, we report the case of a primary meningococcal arthritis without any other typical symptoms of meningococcemia in a young COVID-19 male patient.

\section{Case presentation}

The patient is an 18-year-old man with no medical history, no usual medication nor drug abuse. Familial history revealed a possible psoriasis in the mother, a possible Crohn's disease in the uncle and HenochSchönlein purpura in the sister. There was no relevant traveling exposure, nor any evidence of zoonotic contamination such as tick bites. There was no unprotected sexual exposure, nor any contact with ill people, within the prior weeks. Lastly, no trauma was reported.

On day 1 , the patient consulted his family doctor for fever, asthenia, myalgia, headache, erythema of hands and feet and burning sensation during urination. On day 2 , the retro-transcriptase polymerase chain reaction (RT-PCR) for SARS-CoV-2 was positive, and the patient stayed home awaiting improvement. On day 8 , he presented with a painful and swollen right knee, and a worsening asthenia and fever, but an improvement in respiratory and cutaneous symptoms. On day 14, he consulted a rheumatologist at our hospital in the city of Saint-Quentin, France, for persistent pain in the right knee, but physical examination was strictly normal.

On day 15, the patient was admitted in our Internal Medicine department as signs of arthritis were found upon new examination of his right knee. The other joints were normal, the backbone was painless. Respiratory examination showed full recovery of COVID-19. Cutaneous examination showed livedo reticularis on the feet, petechiae on the bottom-half part of legs and arms. There were no signs of psoriasis. Abdominal examination was normal, no diarrhea was reported. Neurological examination was normal, no meningeal syndrome was found, therefore lumbar puncture was not performed.

At that time, white blood cell count was $8.5 \mathrm{G} / \mathrm{L}$ (Neutrophiles: $6.84 \mathrm{G} / \mathrm{L}$, Lymphocytes: $0.86 \mathrm{G} / \mathrm{L}$, Monocytes: $0.77 \mathrm{G} / \mathrm{L}$ ), C-reactive protein was $142 \mathrm{mg} / \mathrm{L}$. Four blood cultures were performed, each were negative even with extended incubation time. Knee puncture yielded a purulent discharge, no germ nor crystal were spotted upon direct examination. Due to low prevalence of resistant N.meningitidis in France, the patient was first treated with amoxicillin $6 \mathrm{~g}$ /day.

Besides, HIV (human immunodeficiency virus) and HCV (hepatitis C virus) RT-PCR were negative. Syphilis and HBV (hepatitis B virus) serologies were negative. Chlamydia and Gonococcal RT-PCR in urine were negative. The urine culture remained negative. RT-PCR for SARS-CoV-2 was also negative, more than 2 weeks after the onset of first symptoms. At this stage, HenochSchönlein purpura was suspected.

The knee fluid culture was performed according to our hospital standardized protocols upon arrival at the laboratory. Direct examination was performed after Gram and May-Grünwald Giemsa stains but was negative. As for cultures, one run was performed in Schaedler's anaerobe broth for 14 days, two others were performed on blood agar and chocolate agar in aerobic and aero-anaerobic conditions for 10 days. The aerobic culture was positive 2 days later and was identified as Neisseria meningitidis. A sample was then sent to the French National Reference Center at the Pasteur Institute in Paris for N.meningitidis for further testing in an appropriate transport media. Slide agglutination serogrouping led to identify Serogroup B. It was performed, as well as antimicrobial susceptibility testing, in accordance with international guidelines based on the French Microbiology Society and the Clinical and Laboratory Standards Institute guidelines, as described in chapter 6 and 11 of the 2011 World Health Organization "Laboratory Methods for the Diagnosis of Meningitis caused by Neisseria meningitidis" [11]. As such, a $4 \mathrm{~mm}$ depth Mueller-Hinton broth agar plate with 5\% of sheep blood was used to perform a Kirby-Bauer disk diffusion test to evaluate the strain sensitivity to penicillins, cefotaxime, ciprofloxacin, chloramphenicol, rifampicin and azithromycin. Minimal inhibitory concentrations were determined using both disk diffusions and gradient strips in a $5 \% \mathrm{CO} 2$ enhanced atmosphere.

As the strain had intermediate sensitivity to amoxicillin and penicillin $G$, the patient was finally treated with cefotaxime $9 \mathrm{~g} /$ day for 2 weeks and surgical drainage. The drain was removed 2 days after due to absence of fluid and local improvement. Other cases of PMA were treated with similar protocols $[3,5]$. Family and contacts were treated with prophylactic rifampicin for 2 days. 
The patient improved 1 month after the treatment. The knee was painless, without any further impairment.

\section{Discussion and conclusions}

Vaccination coverage for N.meningitidis serogroup $\mathrm{C}$ is low in France and consequently, our patient was not vaccinated. Besides data on the effect of vaccination on PMA is missing, most probably because only few cases are described. The most prevalent serogroup in France is the serogroup B [3], which can explain its presence in our case. This serogroup also fits the nosological frame of chronic meningococcemia associating cutaneous and rheumatologic symptoms mostly, with irregular fever and presence of N.meningitidis in fluids $[4,6]$. No specific N.meningitidis serogroup seems to be associated with a higher probability of PMA [5]. Furthermore, septic arthritis is usually mutilating, for example Staphylococcus aureus or N.meningitidis itself in the nosological frame of invasive meningococcal disease, is associated with a poor outcome in a significant number of cases [1]. However, PMA seems to have a far better outcome than usual septic arthritis, even though serogroup $C$ seems to be less aggressive and associated with an even better outcome [2]. This may suggest that PMA is, for some part, immune-mediated, with the presence of N.meningitidis being the trigger of such immune response, or even being incidental.

The early laboratory analysis showed no sign of immunodeficiency and there is no evidence of any immunocompromising condition in this young patient, in particular no spleen malfunction, nor any complement anomaly. Protein electrophoresis did not show any abnormality except for non-specific inflammation. The familial history of autoimmunity, especially the possible psoriasis in the mother, and a favorable undetermined genetic condition may be an explanation for this peculiar clinical situation, as hypothesised by Lavoipierre et al. in their own case report [5]. On the contrary, the results of Barahona et al. show that there is no higher prevalence in immunocompromised people, neither innate nor adaptive immunodeficiency, as most PMA cases occur in immunocompetent patients [2]. Even though acquired immunodeficiency is more and more prevalent with the ever growing use of immunomodulating treatments, and despite the rise of incidence of immunocompromising conditions such as diabetes, we do not observe any rise in the number of PMA. This may suggest that PMA are not linked to any specific immunodeficiency. On the contrary, we may conclude that PMA are the result of a rare combination of a propitious immune terrain and an acute condition allowing colonizing N.meningitidis to enter the bloodstream, thus strengthening the hypothesis of a dysimmunity-related condition rather than a symptom of actual immunodeficiency.
Saint-Quentin hospital takes care of a large territory as it stands in a mostly rural area. Farmers and hunters constitute a significant part of our patients. Bone and joint symptoms are quite common as traumas are frequent and prevalence of zoonotic diseases presenting with such symptoms is one of the highest in France. Diabetes also has a high frequency in our region, which has already been described as an underlying condition in another PMA case [12] but was not found in our patient. Furthermore, this case took place during the second COVID-19 wave in France, and our hospital was heavily hit and involved in helping our closest large hospitals by admitting a part of their ever growing number of inpatients. Thus, such a patient flow might have facilitated the occurrence of such a rare case.

Jovani et al. and Yokogawa et al. raised concerns about misdiagnosing other classic aetiologies of arthritis, such as crystal arthritis and psoriatic arthritis $[8$, 9]. Our patient did not have any risk factors for crystal arthritis and synovial fluid examination did not find any crystals. With regards to our patient's familial history, a first bout of psoriatic arthritis may be hypothesized, but no skin or nail specific lesions were reported by the patient nor found upon examination and clinical improvement was achieved without any specific treatment for psoriasis.

In conclusion, the main interest of this case is the link between COVID-19 and the onset of arthritis 1 week later. As there is no history of trauma nor any exposure to a confirmed case of meningitis, we may assume a blood-borne infection from meningococcal carriage, even though N.meningitidis was not found in any of the four blood cultures. No immunocompromising condition was found. The pathophysiology of the bloodstream involvement remains unknown [1]. With nasopharyngeal carriage of N.meningitidis being frequent $[1,5]$, we may presume that the impairment of our patient's respiratory tract by the SARS-CoV-2 infection may have facilitated bloodstream involvement and eventually arthritis. Such rare but serious complications of COVID-19 should be investigated, as they may appear later in the course of the disease. Further research is needed to highlight the link between COVID-19 and Neisseria meningitidis arthritis.

\section{Abbreviations \\ PMA: Primary meningococcal arthritis; RT-PCR: Retro-trancriptase, polymerase chain reaction; HIV: Human immunodeficiency virus; HBV: Hepatitis B virus; HCV: Hepatitis C virus; COVID-19: Coronavirus disease, 2019; SARS-CoV- 2: Severe acute respiratory syndrome coronavirus 2}

\section{Acknowledgments \\ Not applicable.}

Authors' contributions

ND and MC contributed equally to this manuscript in conceptualization, writing, editing and reviewing the manuscript, other authors CD, RS, EL and 
BB contributed in editing and reviewing. YD and $\mathrm{HB}$ reviewed and validated this manuscript. All authors read and approved the final manuscript.

\section{Funding}

This research received no specific grant from any funding agency in the public, commercial, or not-for-profit sectors.

\section{Availability of data and materials}

Data sharing is not applicable to this article as no datasets were generated or analysed during the current study.

\section{Declarations}

Ethics approval and consent to participate

Not applicable.

\section{Consent for publication}

The age of majority being 18 in France, written informed consent was obtained from the patient for publication of this case report and any accompanying images. A copy of the written consent is available for review by the Editor-in-Chief of this journal.

\section{Competing interests}

The authors declare that they have no competing interests.

\section{Author details}

${ }^{1}$ Pneumology and Infectious Diseases department, Saint-Quentin hospital, 1 avenue Michel de l'hospital, 02100 Saint-Quentin, France. ${ }^{2}$ Internal Medicine department, Saint-Quentin hospital, 1 avenue Michel de l'hospital, 02100 Saint-Quentin, France.

Received: 15 December 2020 Accepted: 19 May 2021

Published online: 29 May 2021

\section{References}

1. Stinson C, Burman C, Presa J, Abalos M. Atypical presentation of invasive meningococcal disease caused by serogroup W meningococci. Epidemiol Infect. 2020;148:e12. https://doi.org/10.1017/S0950268819002152

2. Barahona M, Catalan J, Sato Y, Hinzpeter J. Primary meningococcal type C arthritis: a case report and literature review. Case Rep Orthop. 2017;2017: 4696014-3. https://doi.org/10.1155/2017/4696014.

3. Gómez BO, Feito CR, Vázquez DG-G, Vega MG, García Viejo MÁ. Primary meningococcal septic arthritis case report and literature review of an unusual manifestation of meningococcal disease. Bull Hosp Jt Dis (2013). 2019;77:140-5

4. Prista-Leão B, Almeida F, Carvalho AC, Silva S, Sarmento A. Chronic meningococcemia. IDCases. 2019;15:e00502. https://doi.org/10.1016/j.idcr.2 019.e00502.

5. Lavoipierre V, Dellyes A, Aubry C, Zandotti C, Lafforgue P, Parola P, et al. Acute polyarthritis in a young patient caused by meningococcal and parvovirus B19 infections: a case report and review of the literature. J Med Case Rep. 2016;10(1):362. https://doi.org/10.1186/s13256-016-1156-4.

6. Brandão AAGS, Prokopowitsch AS, de Campos FPF, Yoshihara LAK. Chronic meningococcemia: a rare presentation of meningococcal disease: case report. Autops Case Rep. 2012;2(1):29-35. https://doi.org/1 0.4322/acr.2012.005

7. Masson-Behar V, Jacquier H, Richette P, Ziza J-M, Zeller V, Rioux C, et al. Arthritis secondary to meningococcal disease: a case series of 7 patients. Medicine (Baltimore). 2017;96(29):e7573. https://doi.org/10.1097/MD. 0000000000007573.

8. Yokogawa N, Minematsu N, Katano H, Suzuki T. Case of acute arthritis following SARS-CoV-2 infection. Ann Rheum Dis. 2020;80(6):e101. https://doi. org/10.1136/annrheumdis-2020-218281.

9. Jovani V, Pascual E, Vela P, Andrés M. Acute arthritis following SARS-CoV-2 infection. J Med Virol. 2020;93(2):661. https://doi.org/10.1002/jmv.26440.

10. López-González M-D-C, Peral-Garrido ML, Calabuig I, Tovar-Sugrañes E, Jovani V, Bernabeu P, et al. Case series of acute arthritis during COVID-19 admission. Ann Rheum Dis. 2020;80(4):e58. https://doi.org/10.1136/a nnrheumdis-2020-217914.

11. World Health Organization, Centers for Disease Control and Prevention (U.S. ). Laboratory methods for the diagnosis of meningitis caused by neisseria meningitidis, Streptococcus pneumoniae, and Haemophilus influenzae: WHO manual 2011.

12. Nihonyanagi S, Sunakawa K, Cui L, Masaki T, Wada T, Hoshiyama T, et al. A very rare case of primary meningococcal arthritis in an adult male. Clin Case Rep. 2015;3(2):76-80. https://doi.org/10.1002/ccr3.151.

\section{Publisher's Note}

Springer Nature remains neutral with regard to jurisdictional claims in published maps and institutional affiliations.
Ready to submit your research? Choose BMC and benefit from:

- fast, convenient online submission

- thorough peer review by experienced researchers in your field

- rapid publication on acceptance

- support for research data, including large and complex data types

- gold Open Access which fosters wider collaboration and increased citations

- maximum visibility for your research: over $100 \mathrm{M}$ website views per year

At $\mathrm{BMC}$, research is always in progress.

Learn more biomedcentral.com/submissions 